\title{
DESIGNING AN INFRASTRUCTURE BY RFID BASED HYBRID TECHNOLOGY SMART WRISTBAND (HTSW) TO ENHANCE THE QUICK RESCUE OPERATION IN INDUSTRIAL ACCIDENT
}

\author{
Md. Kamruzzaman ${ }^{1 *}$, Md. Shariful Islam ${ }^{1 * *}$, Md Zakaria Haider $^{2}$, Asiful Habib ${ }^{3}$ \\ ${ }^{1}$ Institute of Information Technology(IIT), University of Dhaka, Dhaka, Bangladesh \\ *mkzamanmitdu@gmail.com ** shariful.islamegmail.com \\ ${ }^{2}$ Dept. of Electrical \& Electronic Engineering, Bangladesh University of Engineering and \\ Technology(BUET), Bangladesh \\ zakariahaider14@gmail.com \\ ${ }^{3}$ Dept. of Electrical \& Electronic Engineering, Dhaka University of Engineering and \\ Technology(DUET), Gazipur, Bangladesh \\ asifhimulyahoo.com
}

\begin{abstract}
Rescue operation plan playing a vital rule to saving maximum number of life in the time of natural or manmade accident in Industry. Because of high density of labour in industry, possible fatality is increasing day by day. Around 11,000 workers die each year in industrial accidents in Bangladesh. In the last few years, the fastest growing industry of Bangladesh, garments sector has witnessed many industrial accidents specially factory fires and building collapse where many people died and wounded. However, fire service and civil defence has no modern technology to face this kind of big disaster in industry. This paper, firstly discuss about recent industrial disaster in Bangladesh specially garments sector and try to find out the obstacles of quick rescue operation after a disaster occurs. It is important to know how many people are present in a damaged building or structure that needs to evacuate. Radio-frequency identification (RFID) technology is a wireless Automatic Identification and Data Capture (AIDC) technology and could be use in the immediate or aftermath of major disasters. This paper proposed, an infrastructure and a wristband model named "Hybrid Technology Smart Wristband (HTSW)" where RFID, RF-transceiver, Microcontroller and health sense technologies are integrated. In normal condition, HTSW will help the employee management system and in disaster situation, it will enhance the quick rescue operation by identifying the approximate location of dead and live injured person, making plan for transferring injured and trapped victims. In the time of regular and disaster situation, the operation procedures of proposed HTSW infrastructure have explained here.
\end{abstract}

\section{KEYWORDS}

RFID, RF-transceiver, Rescue Operation, Disaster Management, Bangladesh Garments Sector, Hybrid Technology Smart Wristband (HTSW)

\section{INTRODUCTION}

Industries are the backbone of a country's economy. According to International Labour Organization (ILO) around 11,000 workers die each year in industrial accidents in Bangladesh, [1]. Garments are the biggest industry in Bangladesh. An analysis shows that, around $17 \%$ of the country's GDP (Gross Domestic Products) contributed by Garments industry [2] and apparel

DOI : 10.14810/elelij.2015.4102 
industry took the export earnings from USD 31.57 million in 1983 to USD 21.5 billion in 2013 [3] which making Bangladesh the world's second largest apparel producer after China. Bangladesh currently has more than 5600 garment factories where 4.4 million people are employed [3]. In the last decade, Bangladesh garments sector has witnessed many industrial accident specially factory fires and building collapse where many people died and wounded. Since 1990 to 2012, estimated 571 workers were killed by fire accidents and collapse of buildings while between January' 13 to May ' 13 death toll rose to 1150, [4]. Frequent industrial disasters are depleting the image of the sector and keeping the workers scared of life lose while they are working. However, reality is, fire service and civil defence has no modern technology to face this kind of big disaster. This paper illustrates few example of recent disaster in Bangladesh garments sector and tries to find out the obstacles of quick rescue operation after a disaster occurs.

Radio-frequency identification (RFID) technology is a wireless Automatic Identification and Data Capture (AIDC) technology. RFID could be use in the immediate aftermath of major disasters, like an earthquake/fire-hazard, to help save lives. Making an effective rescue plan is the most important issue to save life. When a disaster occurs, it is important to know the number of people trapped in the damaged building or structure that needs to evacuate. In these scenarios, RFID and RF-transceiver technology can facilitate the dispatch of rescue personnel and provide real-time information that could be use to organize search and rescue missions.

In this paper, we proposed an infrastructure and a wristband model named "Hybrid Technology Smart Wristband (HTSW)" where RFID, RF-transceiver, Microcontroller and health sense technologies are integrated. The operation procedure of this proposed infrastructure in the time of normal/regular and disaster situation have discussed here. In this paper, we also illustrate a new technique to avoid collision among the signals transmitting from HTSW.

\section{INDUSTRIAL ACCIDENT IN BANGLADESH}

Highly competitive industry, readymade garments accounts for $45 \%$ of all industrial employment but, given the lack of a safety culture in the country in general, cost-cutting measures often affect the health and safety of the workers. Despite a number of initiatives to curb accidents in the garment industry, there are still a significant number of occurrences in this industry. 24th April 2013, 9am, becoming a brutal incident of history, a nine-story commercial building Rana Plaza collapsed at Savar, Dhaka, Bangladesh and left more than 1134 workers dead, more than hundred missing and many other wounded [5].

More than 3,565 people dead and alive have so far been pulled out of the rubble. The fire service and civil defence had no modern technology to locate the trapped person in that collapse building, which makes the rescue operation, delayed. Shahina, a garment worker, who was pinned in the debris for five long days, was about to be rescued. She was trapped behind a fallen pillar, in a suffocating crevice maybe two feet high. However, the efforts came to 'zero' as fire caught the rescue place [6].

Following another source, Reshma, a garment worker, was found alive under the debris of Rana Plaza at 3:30pm Friday on 17th day of building collapse. Reshma was trapped in the basement of the eight-storey Rana Plaza [7]. The rescue operation was declared finished on the 20th day of the operation. Unfortunately, there is no comprehensive statistics on the status of fire provisions and management practices in the manufacturing factories in Bangladesh. 
Electrical and Electronics Engineering: An International Journal (ELELIJ) Vol 4, No 1, February 2015

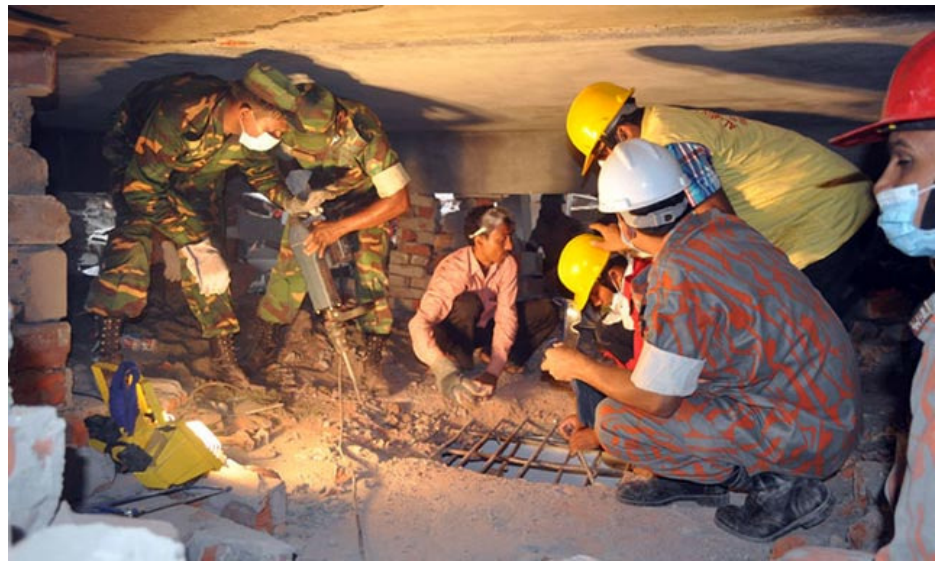

Figure 1. Finding the injured person by traditional rescue operation at Rana Plaza, Savar, 26 April 2013.

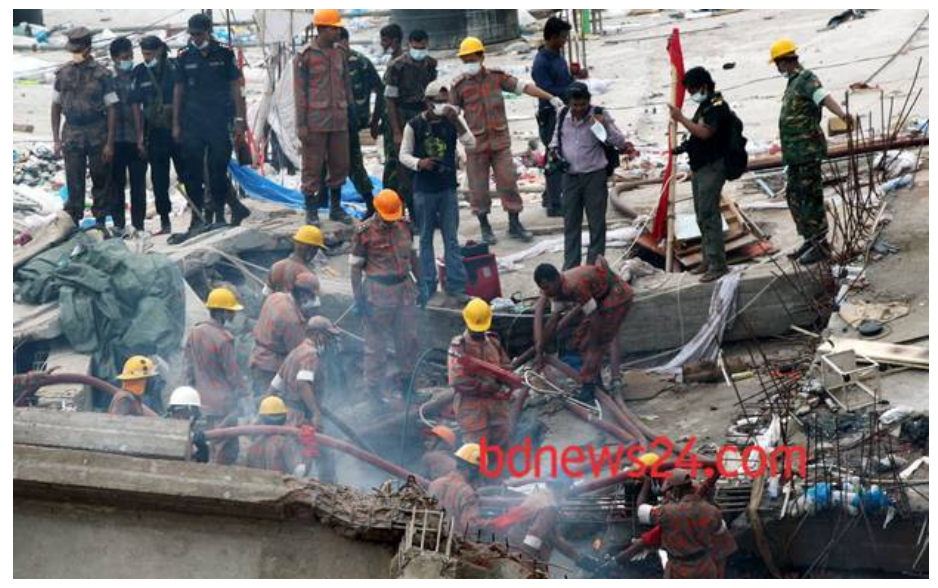

Figure 2. After 5 long days, rescue team found a garments worker alive named Shahina, but failed to rescue her alive.(Photo: bdnews24.com)

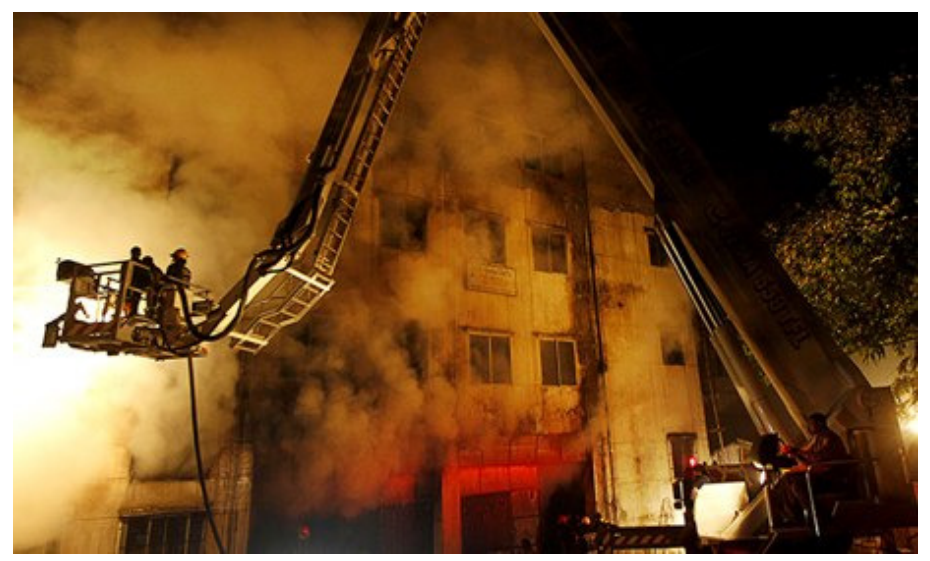

Figure 3. Bangladeshi fire fighters battle a fire at Tazreen garment factory in Savar, Dhaka. (Photo: The Guardian)

A fire broke out at Tazreen Fashions, Nischintapur Ashulia Dhaka, Bangladesh on the night of November 24, 2012. At least 117 people were confirmed dead in the fire, and at least 200 were 
injured [8]. There was no technology application that can help to find the trapped workers immediately and make an effective rescue plan, which could save more life.

On 11th April 2005, an 8-storey industrial building situated in Savar industrial belt collapsed which killed 64 workers and critically injured several hundreds. Several of them have become physically disable [4]. Shahriar Fabrics Industries Ltd. and Spectrum Sweater Factories were housed in that building. Along with industrial growth, the industrial accidents have also increased tremendously. According to the survey report of Bangladesh Garment Manufacturers and Exporters Association (BGMEA), 47 fire incidents occurs from 2006 to January 2010, [4]. The following Figure. 4 is generated with data collected from different liable sources.

- Died workers =Critically Injured

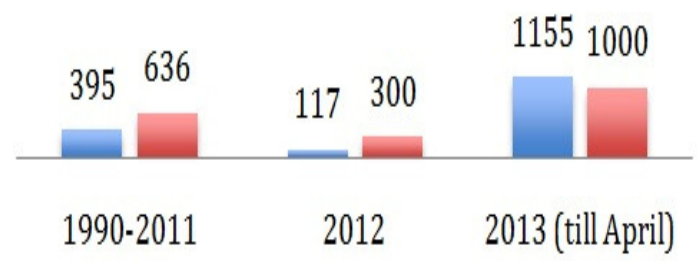

Figure 4. Causalities in Clothing Sector in 1990-2011, 2012 and 2013, [4]

\section{Present Scenario of RFID Technology in BangladeSh}

A little area of both the private and public sectors are driving the RFID market in Bangladesh [9]. Deltech Ltd., a global RFID solution provider has already helped several organizations implement RFID [10]. According to Mr. Delwar Hossain, founder of the Deltech, hands-free ac- cess control and employee-tracking systems, as well as asset management are the most popular applications businesses are asking for. Appollo Hospital, Dhaka has completed the first phase of an RFID project to track employee attendance as well as assets [10]. The Bangladesh Army also plans to employ this technology to track soldiers and visitors entering its Dhaka Cantonment as well as track retail purchases. In many export sectors of Bangladesh ranging from garments to household and office furniture, large international buyers can get rid of the age-old barcode-based product identification systems for RFID tags to reduce product tracking and inventory management costs of the items, which will ultimately help the country to be competitive in the world market. US retailers like Target, Wal-Mart, etc. are major buyers of Bangladesh's garment products. Since Wal-Mart has already announced its plans to require majority of their suppliers to implement RFID based tracking systems [9]. The sea change in global integrated supply chain management necessitates a rapid mobilization of our government, IT and export industry leaders and experts to devise a uniform RFID strategy for Bangladesh.

\section{RFID TECHNOLOGY}

Radio Frequency Identification (RFID) is an automated data collection technology that enables equipment to read tags attached to the objects without contact or line of sight. RFID uses radio frequency (RF) waves to transfer data between a reader and an item to identify, track or locate the item. According to the European e-Business Watch large-scale survey [11] of RFID adoption strategies and impacts in four broad economic sectors, $14 \%$ of the European companies interviewed were piloting, using or implementing RFID technology in 2007. Companies that were using RFID or planning to use RFID expected major effects on: a) inventory management (49\% of companies using or planning to use RFID), b) control and efficiency of in- bound logistics 
(46\%), and c) merchandise management and reduced out-of-stocks (44\%). The major costs for those using or planning to use the technology were seen to be the costs of project implementation and system integration (39\% of all companies using or planning to use RFID) [9].

The RFID system consists of a tag, which is made up of a microchip with a coiled antenna, an interrogator or reader with an antenna and a middleware application that is integrated into a host system. The RFID market will generate USD70.5 Billion from 2012 to the end of 2017 and 50 Million electronic devices will be RFID enabled in 2012[12]. An RFID system has several features and functions that depend on the frequency band, nature of the power source, tags, etc.

\subsection{RFID Tag}

RFID tags, also known as transponders, contain a chip and an antenna. The latter enables the chip to respond to an interrogation signal transmitted from the RFID reader. RFID tags can be attached to or embedded in a physical object to be identified. They store product-item information such as manufacturer, product lot, size and category, production date, expiration date, final destination, etc. RFID tags have various characteristics such as designs, power source, carrier frequency, communication method, read range, data storage capacity, memory type, size, operational life, and cost. Figure 5 shows different types of RFID tag and Table- 1 shows the frequency range and transmission range $\&$ speed of different types of RFID tag.

(a)

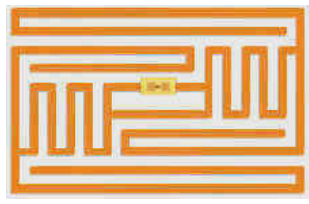

(b)

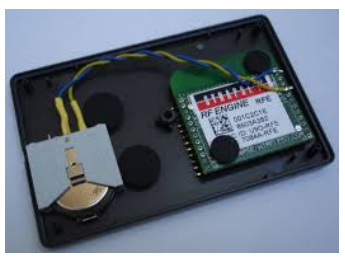

(c)

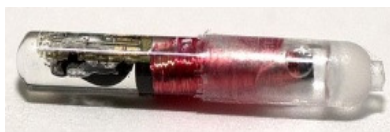

Figure 5. Different types of RFID tag:- (a) A passive RFID tag (from [Wiki-RFID], used under the GNU Free Documentation License), (b)Active RFID Tag, (c) RFID Microchip for implant.

Table 1. RFID Tag Frequencies [13]

\begin{tabular}{|l|l|l|l|}
\hline Band & Regulations & Range & Data speed \\
\hline $120-150 \mathrm{kHz}(\mathrm{LF})$ & Unregulated & $10 \mathrm{~cm}$ & Low \\
\hline $13.56 \mathrm{MHz}(\mathrm{HF})$ & ISM band worldwide & $10 \mathrm{~cm}-1 \mathrm{~m}$ & Low to moderate \\
\hline $433 \mathrm{MHz}(\mathrm{UHF})$ & Short Range Devices & $1-100 \mathrm{~m}$ & Moderate \\
\hline $\begin{array}{l}300-1000 \mathrm{MHz}(\text { Bangladesh) } \\
865-868 \mathrm{MHz} \text { (Europe) } \\
902-928 \mathrm{MHz} \text { (N. America) UHF }\end{array}$ & ISM band & $1-12 \mathrm{~m}$ & Moderate to high \\
\hline $2450-5800 \mathrm{MHz}$ (microwave) & ISM band & $1-2 \mathrm{~m}$ & High \\
\hline $3.1-10 \mathrm{GHz}$ (microwave) & Ultra wide band & $1-200 \mathrm{~m}$ & High \\
\hline
\end{tabular}

\subsection{RFID Reader Writer}

RFID readers, also known as interrogators, are electronic devices that emit and receive radio signals through the antennas coupled to them. RFID readers capture data stored in RFID tags and, depending on the technology used, they may also overwrite data on the tags. Readers are responsible for the information flow between the tags and the host system via the RFID middleware. Furthermore, they are able to identify and read a large number of tags per second without any problem. Figure 6 shows different types of RFID reader. 
(a)

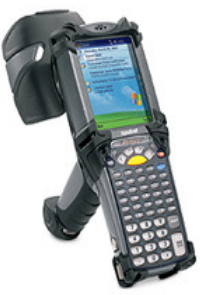

(b)

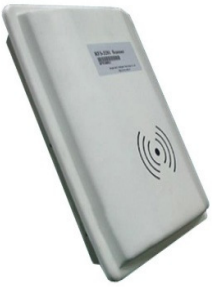

(c)

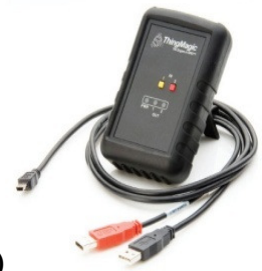

(d)

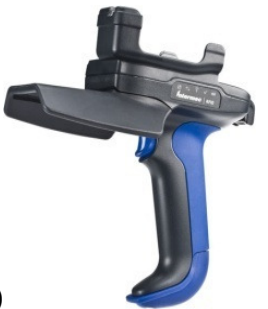

Figure 6. Different types of RFID Reader : (a) Handheld RFID reader, (b) Fixed RFID reader, (c) USB RFID reader, (d) Bluetooth RFID reader.

\subsection{Antennas and Radio}

The RFID physical layer consists of the actual radios and antennas used to couple the reader to the tag so that information can be transferred between the two. Radio energy is measured by two fundamental characteristics: the frequencies at which it oscillates and the strength or power of those oscillations. Commercial FM broadcast stations in the Bangladesh transmit with energy at a frequency between $88 \mathrm{MHz}$ and $108 \mathrm{MHz}$, or 1 million isolations per second. The AM spectrum, by contrast, transmits at 500,000 to $1,500,000$ oscillations per second, or between $500 \mathrm{KHz}$ and $1500 \mathrm{KHz}$. Microwave ovens cook with RF energy that vibrates 2.4 billion times each second, which is $2.4 \mathrm{GHz}$.

(a)

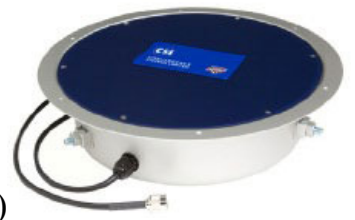

(b)

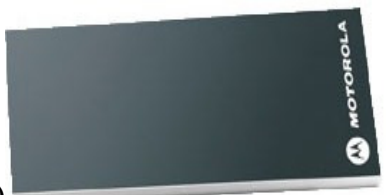

(b) Far Field

RFID systems based on LF and HF frequencies make use of near field communication and the physical property of inductive coupling from a magnetic field. The reader creates a magnetic field between the reader and the tag and this induces an electric current in the tag's antenna, which is used to power the integrated circuit and obtain the ID. The ID is communicated back to the reader by varying the load on the antenna's coil, which changes the current drawn on the reader's communication coil; further detail of the physics of the operation can be found in ACM Queue's RFID special edition [14]. RFID systems based on UHF and higher frequencies use far field communication and the physical property of backscattering or 'reflected' power. Far field communication is based on electric radio waves: the reader sends a continuous base signal frequency that is reflected back by the tag's antenna. During the process, the tag encodes the signal to be reflected with the information from the tag (the ID) using a technique called modulation (i.e. shifting the amplitude or phase of the waves returned).

\subsection{RFID Middleware}

The RFID middleware is at the core of any RFID system. It is responsible for monitoring readers, managing, filtering, processing and aggregating all the data collected from products by readers and then routing the data to the dedicated information systems. In addition, the RFID middleware can be used to manage and control RFID readers' infrastructure. It can be considered as the nervous system of RFID technology since it provides key functionalities, such as the efficient management of the data produced by the RFID system. 


\subsection{Coupling}

While it is possible to build RFID systems such that both the tag and reader contain a radio transmitter and a radio receiver, this method of operation is ideal only for active systems attempting to communicate over the longest distances. In the near field, a tag couples with a reader via electromagnetic inductance. The antennas of both the reader and the tag are formed as coils, using many turns of small gauge wire. The current in the reader's coil creates a magnetic field. This field, in turn, induces a current in the coil of the tag. A transformer works by the same principle, and in essence, the coils of the reader and tag together form a transformer. The reader communicates with the tag by modulating a carrier wave, which it does by varying the amplitude, phase, or frequency of the carrier, depending on the design of the RFID system in question. This modulation can be directly detected as current changes in the coil of the tag. The tag communicates with the reader by varying how much it loads its antenna. This in turn affects the voltage across the reader's antenna. By switching the load on and off rapidly, the tag can establish its own carrier frequency (really a subcarrier) that the tag can in turn modulate to communicate its reply.

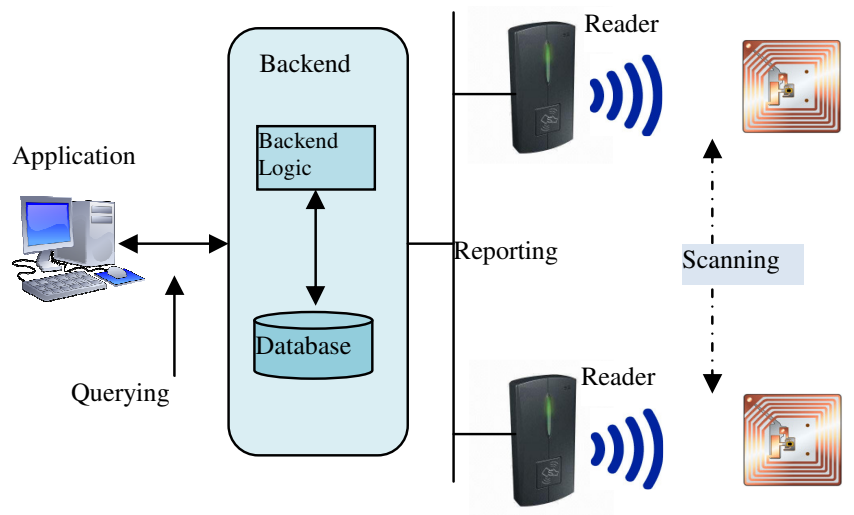

Figure 8. Basic layout of RFID communication system

In general, a RFID system has a structure as depicted in figure 8. RFID readers scan tags, and then forward the information to the backend. The backend in general consists of a database and a well-defined application interface. When the backend receives new information, it adds it to the database and if needed performs some computation on related fields. The application retrieves data from the backend. In many cases, the application is collocated with the reader itself.

\section{INTEGRATION OF RFID AND SENSOR NETWORK}

RFID and sensor networks are both important components of pervasive computing. Sensor network is usually used to sense and monitor physical, chemical, and biological environments through sensing of sound, temperature, light, etc. Any object that is embedded with an RFID tag can be track-able or sensible in typical application of an RFID system. Although RFID technology has limitations such as low tolerance to fluid or metal environments, it can extend the ability of a sensor network by providing sensible property to otherwise insensible objects.

On the other hand, wireless sensor networks offer a number of advantages over traditional RFID implementations. First, sensors are able to provide much more information, such as the measurement of temperature, humidity, pressure, vibration intensity, sound intensity, power-line voltage, chemical concentrations, pollutant levels, etc., than simple RFID Second, incorporation with sensors enables RFID to push logic into nodes to enable RFID readers/tags to have 
intelligence. This allows for new applications, such as real-time warning of proximity to hazardous substances or enforcement of safety rules in the operation of heavy machinery.

\subsection{Integrating Tags with Wireless Sensor Nodes}

RFID tags integrated with sensors have limited communication capabilities. In high-end applications, it is possible to add RFID tag's capabilities to wireless sensor nodes. These new nodes may be compliant with existing RFID standards or they can have proprietary protocols. CoBIs RFID tags [15] are designed to monitor the ambient conditions around them and provide alerts when detected conditions break predetermined business rules. Each CoBIs tag carries an accelerometer (movement) sensor, a wireless transceiver, up to 10 kilobytes of memory, and other computing components for storing and processing business rules. The tags are able to communicate with each other via a proprietary peer-to-peer protocol. Each node transmits not only its unique ID number but also details of its sensed data to all other nodes within a 3-meter range.

\subsection{Integrating RFID Readers with Wireless Sensor Nodes and Wireless Devices}

Another type of integration of RFID and sensors is to combine RFID readers with RF transceiver. Integration of the RFID readers with wireless sensor nodes and wireless devices enables new functionalities and opens the door to a number of new applications. Wireless devices will be able to sense environmental conditions, read identification numbers from tagged objects and effectively transmit this information to the host.

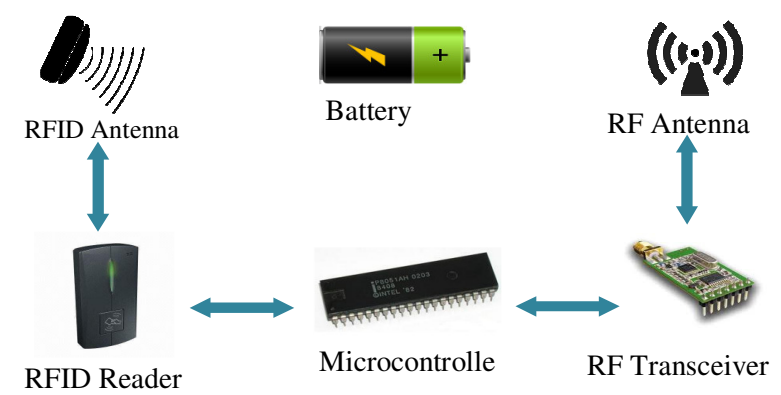

Figure 9. Data flow process to integrate RFID reader and RF transceiver [15]

There are some industry solutions for RFID readers that are equipped with WLAN. One example is ALR-9770 series multi-protocol RFID reader that was developed by Alien Technology. The reader is equipped with up to 4 antenna sets for reliable tag reading and is able to communicate via $802.11 \mathrm{~b} / \mathrm{g}$ standard [16]. In recent years, RFID is more and more used in positioning, tracking and analysis system for moving objects. It reads the information of mobile tags within the effective range by fixed readers to identify and locate moving objects [17].

\subsection{Hybrid Technology Smart Wristband (HTSW)}

HTSW, a RFID based wristband is designed for garments workers. It will be mandatory to wear this band in hand when they stay in garments premises. This band consists by the integrated technology of RFID tag, Health sensor and RF-transceiver and Microcontroller. 


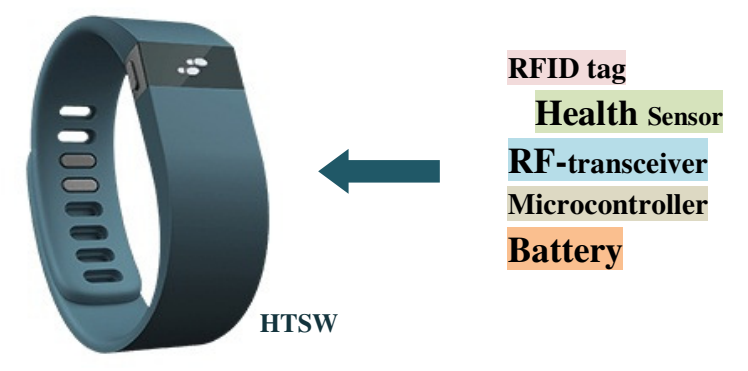

Figure 10. Proposed Hybrid Technology Smart Wristband (HTSW)

In our proposed HTSW model, we have used short-range low power consuming passive RFID tags, which will be used to monitor some basic activities of the garments workers (i.e. auto checkin / checkout, attendance management etc). The RF-transceiver will transmit two types of information that is fixed and variable. The fixed data are employee name, ID and blood group; those are provided by RFID passive tag and variable data that is employee heartbeat provided by pulse sensors. We have used a simple microcontroller to store data and to combine all the works done by the HTSW properly.

\section{Regular OPERATION AND RESCUE OPERATION}

In our proposed infrastructure, there are three basic systems 1) HTSW at human hand, 2) central wired/wireless network at factory/office and 3) external universal RF-locator for the rescue team. In normal condition, HTSW will help the employee management system and in disaster condition, it will enhance the quick rescue operation. The behaviour of HTSW will depends on the working premises and the central network condition.

\subsection{Operation in general condition}

Possible task in garments premises

- Auto check-in to central interface by RFID

- Attendance Management

- Employee monitoring

- Appling restricted entry in particular area.

- Over-time management

- Employee Health monitoring

Out of garments premises

- Auto checkout from central interface by RFID

-After successful checkout by acknowledgement, HTSW will move in auto shutdown for fixed duration like 10/12 hours to save the battery power. In addition, there will be an option to switch on HTSW manually. 


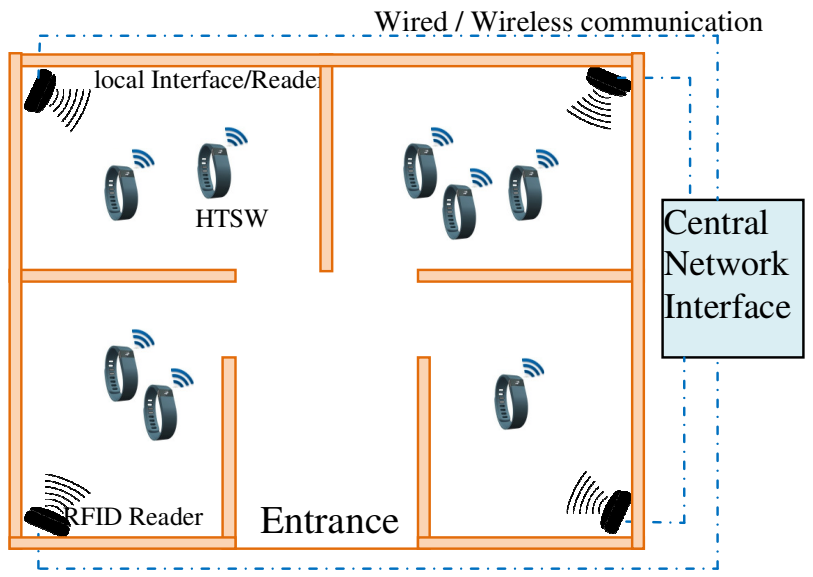

Figure 11. Communications among HTSW, local interface and central network interface at the time of regular operation.

\subsection{Operation in disaster (Fire/Building-collapse/Earthquake)}

The central network will be connected with multiple sensors for detecting disasters like fire hazards, earthquake etc. After detecting any sign of disasters, the central network will inform the local networks and the local networks will inform all the HTSW to switch to emergency mode. If the networks fail to do it then the HTSW will automatically switch to emergency mode after not receiving any signal from the local networks for a certain amount of time .After entering this mode each HTSW will begin to transmit signals at regular intervals. Every time each wristband will transmit several bytes of data in different stages.

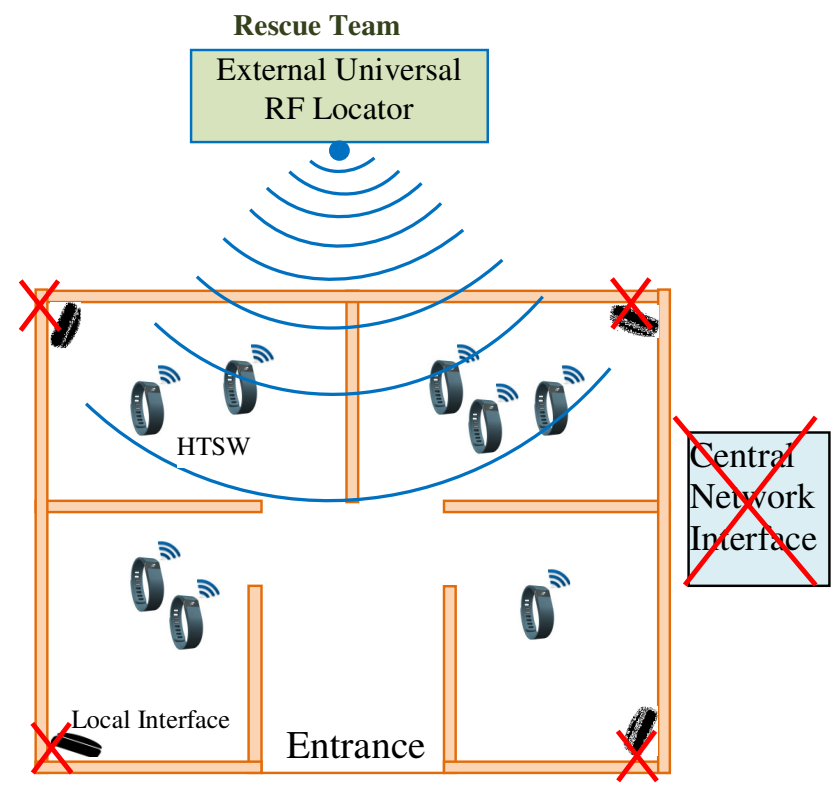

Figure 12. Communications between HTSW and External Universal RF Locator at the time of the disaster where, local interface and central network interface both are dead.

First Step: It will send an arbitrary value like a single character or any number so that the receiver can understand the data are coming from a wristband instead of noise. 
Second Step: In this step, it will send its unique ID number. The format will be "AABBBCC", where $\mathrm{AA}$ is the group number $\mathrm{BBB}$ is the unique id number and $\mathrm{CC}$ is the number of people in that group. We have included this information so that when any external locator detects this signal will get an idea approximately how many people are there.

Third Step: In this stage, the wristband will transmit the heart beat data of that individual worker from the pulse sensors so that the physical condition of that worker can be known from the outside at the time of disasters.

Fourth Step: It will send the same arbitrary value, which was send at the very first step just to make sure the end of transmission.

\subsection{Radio Frequency Collision Handling}

In RFID systems with one reader and many tags, tag-to- tag collisions occur when multiple tags transmit their signals simultaneously to the reader. This problem is called tag collision and many tags anti-collision protocols have been proposed in the literature [18]. Many efficient anticollision protocol adopted by different body standards [19] and commercial products are based on the classical Framed-Slotted Aloha. In framed slotted aloha based anti-collision protocols, the reader begins each interrogation round by informing all tags about the current frame size in terms of time slots. Each tag then selects a random time slot and sends its identifier in that timeslot. The probability of tag collision depends on the frame size and the number of tags. Since not all tags can be identified in the one round, the reader repeats the interrogation rounds until all tags are identified [20].

It will be difficult for the external RF locator to detect all the signals at a time. Thus, anticollision algorithms that provide quick and correct recognition regardless of the occurrence of collisions are required. There is much published research on improving probabilistic and deterministic algorithms in different ways. Some works try to improve the way of choosing an optimal frame size by finding an appropriate method of estimating the number of tags in the interrogation zone [21].

In this paper, we propose a new technique to avoid collision among the signals transmitting from HTSW. First, we divide all the HTSW in to several groups. Each group has their unique radio frequency thus all the trans-receivers of the same group contain same frequency. Besides, each wristband will contain its own information along with all the serial numbers of its group mates stored in the internal memory of its own microcontroller.

At the time of disaster when the transmission begins instead of all HTSW one member from each group will begin to transmit signals at a time. When one member of a group finishes its transmission then another member of the same group will start its transmission. Emphasize has given in the synchronizing procedure of transmission so that not more than one member from the same group will start to transmit at a time. This system has certain advantages as each group has different frequency of its own so there is little possibility of overlapping or collision among the signals coming from different groups at a time.

Besides one member from each group transmits signal at a time so there is no possibility of overlapping signals of same frequency. This method is reliable and fast besides it will be easy to implement. Besides, we do not need any complex anti-collision algorithm to avoid signal collision. Another side, each HTSW device will communicate with other nearest HTSW device via RF-transceiver. This inter communication can help the rescue team finding all HTSW of a common network through a single node. As each HTSW will transmit all the identification 
numbers of its group-mates along with its own detecting any signal from any HTSW we can able to approximate the location of the other HTSW of the same group.

\section{Conclusions}

Although, we design the HTSW infrastructure to use in garments factory but this technology can be used in every type of industry and corporate office to enhance the rescue operation in any natural or casual accident. In this paper, a basic idea on quick rescue operation is developed and proposed for high-density lobar industries in Bangladesh. The proposed infrastructure based on RFID technology, Hybrid Technology Smart Wristband (HTSW) could help the rescue team to identify the approximate location $\&$ number of dead \& live injured person and making plan for transferring injured and trapped victims. Although it is our conclusion that the idea of HTSW is feasible, there are many issues to resolve before any full-fledged deployment. To perform more effective and realistic rescue solution, it is necessary to perform further development of proposed system. As future work, some of the issues will be investigated like, (1)designing a strong \& lightweight body for HTSW, (2)developing a scheme to combine an RFID tag/reader, RFtransceiver, and latest health sensor device to produce consistent and accurate victim information, (3)developing a tag database management algorithm and program for central network, (4)designing the universal RF-locator which can locate the HTSW in any hazarded environment and (5)cost \& performance analysis of HTSW infrastructure. Bangladesh government, garments/corporate-office/industry owners and fire service \& civil society, everyone could work jointly to ensure secure environment in industry/office by employing this proposed infrastructure.

\section{REFERENCES}

[1] "Safety and health at Work". International Labour Organization. Available at : http://www.ilo.org/dhaka/Areasofwork/safety-and-health-at-work/lang--en/index.htm

[2] BBC News, "Bangladesh textile workers deaths avoidable" 26 April 2013, Available at : http://www.bbc.com/news/business-22296645.

[3] Bangladesh Garment Manufacturers and Exporters Association (BGMEA), Available at : http://www.bgmea.com.bd/blog/story/2

[4] Clothing Sector gets another death toll, , Cover Story, Bangladesh Textile Today Magazine, Issue: May-2013, Available at : http://www.textiletoday.com.bd/magazine/639

[5] Article:- Death of A Thousand Dreams, Available at : http://www.taslimaakhter.com/rana_plaza_collapse

[6] bdnews24 News : Shahina comes out dead, Available at : http://bdnews24.com/bangladesh/2013/04/29/shahina-comes-out-dead

[7] Report, Brief History of the Rana Plaza Tragedy, Available at: http://news.priyo.com/2013/05/14/end-savar-battle.html

[8] Farid Ahmed (25 November 2012). "At least 117 killed in fire at Bangladeshi clothing factory". CNN. Archived from the original on 25 November 2012. Retrieved 25 November 2012.

[9] A. Masum, F. Bhuiyan and K. Azam, "RFID Applications: Prospects and Challenges in Bangladesh," Journal of Information Security, Vol. 4 No. 2, 2013, pp. 73-79. doi: 10.4236/jis.2013.42009.

[10] S. B. Miles, S. E. Sarma and J. R. Williams, "RFID Tech- nology and Applications," Cambridge University Press, Cambridge, 2008. doi:10.1017/CBO9780511541155

[11] M. L. Chuang and W. H. Shaw, "RFID: Integration Stages in Supply Chain Management," IEEE Engineering Management Review, Vol. 35, No. 2, 2007, pp. 80-87. doi:10.1109/EMR.2007.899757

[12] The Future of RFID - Infographic , Available at : http://rfid.thingmagic.com/rfid-blog/bid/87674/

[13] Wikipedia, Radio-frequency identification, Available at : http://en.wikipedia.org/wiki/Radiofrequency_identification

[14] WANT, R. 2004. The magic of RFID. ACM Queue, vol. 2, no. 7 (October). Association for Computing Machinery. available online at: http://www.acmqueue.com/modules.php?name=Content $\&$ pa=showpage $\&$ pid=216 
[15] Hai Liu, Miodrag Bolic, Amiya Nayak, Ivan Stojmenović, "Integration of RFID and Wireless Sensor Networks".

[16] Alien Technology, ALR-9770 Series Multi-protocol, RFID Readers, Available at: http://store.trustrfid.com/ProductsURL/alien/alr9774.pdf.

[17] Y. Liu, H. Zhang and Y. Wang, "RFID Complex Event Processing: Applications in Real-Time Locating System," International Journal of Intelligence Science, Vol. 2 No. 4A, 2012, pp. 160-165. doi: 10.4236/ijis.2012.224021.

[18] D. Shih, P. Sun, D. Yen and S. Huang, "Taxonomy and Survey of Rfid Anticollision Protocols," Computer Com- munications 2006, Vol. 29, 2006, pp. 2150-2166.

[19] "Epc Global Class 1 Generation 2 Uhf," http://www.epcglobalinc.org

[20] Felemban, E. (2014) Performance Analysis of RFID Framed Slotted Aloha Anti-Collision Protocol. Journal of Computer and Communications, 2, 13-18. doi: 10.4236/jcc.2014.21003.

[21] Majid Alotaibi, Adam Postula, Marius Portmann," Tag Anti-collision Algorithms in RFID Systems a New Trend.", WSEAS TRANSACTIONS on COMMUNICATIONS, Issue 12, Volume 8, December 2009.

\section{Authors}

Md. Kamruzzaman obtained his B. Sc. Engg. Degree in Electrical and Electronic Engineering from the Ahsanullah University of Science and Technology (AUST), in 2009. At present, he is continuing his postgraduate study in Masters of Information Technology under the Institute of Information \& Technology (IIT) in University of Dhaka (DU), Bangladesh. He is currently working as a Sr. Lecturer of Electrical and Electronic Engineering department at Atish Dipankar University of Science and Technology (ADUST), Dhaka, Bangladesh. His research interests include RF Engineering, Wireless Communication, Coal Gasification, Power System and Renewable Energy. Kamruzzaman is a member of Institution of Engineers of Bangladesh (IEB) and IEEE.

Md. Shariful Islam received his B.Sc. and M.Sc. degree in Computer Science from the University of Dhaka, Bangladesh, in the year 2000 and 2002, respectively. He completed his M.S degree in Information Technology from the Royal Institute of Technology (KTH), Sweden, in 2005. He obtained his Ph.D degree in Computer Engineering from Kyung Hee University, South Korea in February 2011. He is now working as an Associate Professor in the Institute of Information Technology (IIT), University of Dhaka, Bangladesh. His current research interests include the design of routing protocols, metrics and MAC protocols for wireless mesh networks. He also worked on security issues related to Wireless AdHoc and Mesh Networks. He has

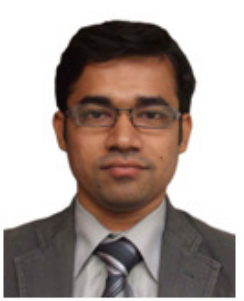
published a good number of research papers in international conferences and journals. He is a member of IEEE and KICS.

Md Zakaria Haider was born in Bangladesh on December 1992. He is third year student in department of Electrical \& Electronic Engineering in Bangladesh University of Engineering and Technology (BUET), Bangladesh. His research interests are in wireless communication system, applications of information theory and coding to modern communication systems.

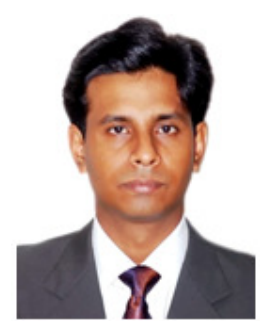

Asiful Habib was born in Dhaka, Bangladesh on $10^{\text {th }}$ November 1987. Currently he is pursuing his postgraduate study under the department of Electrical \& Electronic Engineering (EEE) in Dhaka University of Engineering and Technology (DUET), Bangladesh. He obtained his B. Sc Engineering Degree in Electrical and Electronic Engineering from Ahsanullah University of Science \& Technology in 2009. His research interests include Radio Frequency Engineering, Power System and Renewable Energy.
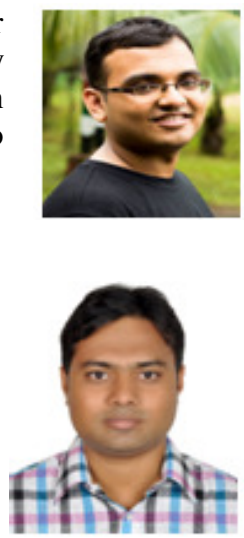